\title{
Caudad Insertion of Pedicle Screws Facilitates Interbody Distraction During Spondylolisthetic Vertebrae Restoration: A Retrospective Study
}

\author{
Xin Xi $\cdot$ Zhili Zeng $\cdot$ Fuping Li $\cdot$ Cong Wang $\cdot$ Bin Ma $\cdot$ \\ Ning Xie · Tsung-Yuan Tsai · Guoan Li · Yan Yu (D) - Liming Cheng
}

Received: July 13, 2021 / Accepted: August 27, 2021 / Published online: September 15, 2021

(C) The Author(s) 2021

\begin{abstract}
Introduction: Based on the results of long-term clinical and radiological follow-up studies of decompression and fusion with internal fixation for degenerative lumbar spondylolisthesis (DLS), we recognized that the direction of the
\end{abstract}

Xin Xi and Zhili Zeng contributed equally to this work and are co-first authors.

X. Xi · Z. Zeng · B. Ma · N. Xie · Y. Yu $(\bowtie)$.

L. Cheng

Department of Spine Surgery, Tongji Hospital, Tongji University School of Medicine, Shanghai 200065, China

e-mail: yyu15@tongji.edu.cn

X. Xi · Z. Zeng · B. Ma · N. Xie · Y. Yu · L. Cheng Key Laboratory of Spine and Spinal Cord Injury Repair and Regeneration, Ministry of Education, Tongji Hospital, Tongji University School of Medicine, Shanghai 200065, China

F. Li

Department of Spine Surgery, Shanghai Fourth People's Hospital Affiliated to Tongji University School of Medicine, Shanghai 200434, China

C. Wang · T.-Y. Tsai

School of Biomedical Engineering, Shanghai Jiao Tong University, Shanghai 200030, China

G. Li

Department of Orthopedic Surgery, Orthopaedic Bioengineering Research Center, Newton-Wellesley Hospital/Harvard Medical School, Wellesley, MA, USA pedicle screw affects the stability of the fixation. However, few studies have analyzed the role of pedicle screw insertion trajectory in disc height recovery after fusion. We therefore analyzed patients' general information, clinical efficacy and sagittal, coronal and implant parameters to determine whether there is a correlation between the insertion trajectory of screws and the recovery of intervertebral space height, with the ultimate aim to provide a basis for improving the clinical efficacy and radiological outcomes of patients with DLS and to identify an optimal technique for spine surgeons that would benefit patients with spondylolisthesis.

Methods: From May 2015 to October 2019, patients who underwent single-segment decompression and fusion with internal fixation for DLS at our department were screened for enrollment in the study. The clinical history, pre- and post-operative lumbar sagittal parameters, intervertebral height, rate of recovery from spondylolisthesis and pedicle screw angle of inpatients were recorded and followed up for at least 6 months. Clinical assessments included the Oswestry Disability Index (ODI) and the Visual Analogue Scale (VAS) for lower back and leg pain. Data on screw angle, fusion segment intervertebral space height and clinical outcome were the primary outputs. Pearson correlation and multivariate regression analyses were performed to investigate the relationship between the pedicle screw angle, the sagittal 
parameters of the fusion segment and clinical efficacy.

Results: A total of 50 patients were initially enrolled, two patients were lost to follow-up after 6 months, 48 patients ( 17 men, 31 women) were eventually enrolled, and the follow-up rate was $96 \%$. At least 6 months after the operation, vertebral spondylolisthesis improved to varying degrees [> 80\% in 17 cases (35.4\%) and $>20 \%$ in 43 cases $(87.5 \%)$, respectively]. Changes in disc height (DH) were significantly associated with lower pedicle screw angle, while lumbar lordosis and segment lordosis remained the same. Multivariate regression analysis showed a significant negative correlation between the upper and lower pedicle screw angles and the change in DH $(P<0.05)$. At 2 weeks post-operation, the VAS score for low back pain and the ODI had improved significantly compared to pre-operation $(P<0.05)$.

Conclusions: These results suggest that the Caudad insertion trajectory technique of pedicle screws may be an ideal alternative for the treatment of DLS.

Trial Registration Number: Chinese Clinical Trial Registry (ChiCTR): ChiCTR1800020368.

Keywords: Lumbar spondylolisthesis; Interbody fusion; Pedicle screw fixation; Caudad insertion trajectory; Intervertebral disc height

\section{Key Summary Points}

Why carry out this study?

Degenerative lumbar spondylolisthesis (DLS) is one of the most common degenerative diseases of the lumbar spine.

DLS causes not only a series of clinical symptoms in patients, but its high incidence also increases the economic and medical burden of individuals, families and governments.
We investigated whether there is a correlation between the insertion trajectory of screws and the recovery of intervertebral space height in patients who underwent single-segment decompression and fusion with internal fixation for DLS, which may ultimately help surgeons to develop an optimal technique for patients with spondylolisthesis.

\section{What was learned from the study?}

Caudad insertion trajectory technique of pedicle screws may be an ideal alternative for the treatment of DLS.

\section{INTRODUCTION}

The most common changes in degenerative lumbar spondylolisthesis (DLS) include anterior vertebral sliding, reduced segmental lordosis angle and loss of intervertebral space height. In the surgical treatment of this condition, the reduction of spondylolisthesis and the degree of reduction are not directly related to the clinical prognosis; rather, the focus is on the reconstruction of lumbar stability through fusion [1-3]. In general, a successful outcome is largely dependent on obtaining a solid fusion, as well as recreating the normal disc height (DH) [4]. Satisfactory bone fusion can effectively prevent post-operative cage shifting and post-operative intervertebral space height loss. Bourghli et al. showed that a restored spinal sagittal balance was correlated with good clinical outcomes following surgery for low-grade spondylolisthesis $[5,6]$. Correcting spondylolisthesis is desirable for effective indirect decompression of the neuroforamina and restoration of spinal sagittal balance. Although optimal methods for operative treatment remain poorly established, there has been a trend toward the use of the interbody fusion technique, which was developed to provide solid fixation of spinal segments while restoring a proper $\mathrm{DH}$ and sagittal balance [7]. 
Pedicle screw fixation is commonly used to facilitate fusion as the screws are believed to help temporarily fix the lumbar spine, obtain good intervertebral DH and restore the segmental lordosis angle. Moreover, the additional stability of pedicle screw fixation provides significantly improved fusion rates [8]. However, a typical complication associated with pedicle screw fixation is screw loosening, which occurs in $0.8-27 \%$ of cases, with the rate potentially exceeding $50 \%$ in patients with osteoporosis [9-12]. In the biomechanical context, injury of the superior adjacent facet joint (FJ) by the pedicle screw increases the axial rotation angle, FJ pressure and pressure within the intervertebral disc $[13,14]$, thus affecting the stability of the adjacent segments.

Studies have shown that bone radiodensity, length of fixation and applied surgical technique, including fusion type, affect complication rates [15]. More recently, a "rocking" technique and a modified version termed the "swing" technique have been developed and brought into regular use. These two techniques are safe and effective reduction methods for spondylolisthesis that not only alleviate symptoms but also achieve a nearly complete reduction of slippage. A study on the stability of pedicle screw fixation in osteoporotic lumbar vertebrae showed that the direction of the screw affected the stability of the fixation [16]. For patients with osteoporosis, the commonly used technique of parallel screws to the upper endplate does not provide the best biomechanical stability [16]. However, few studies have analyzed the role of pedicle screw insertion trajectory in DH recovery after fusion. Also, it is not clear whether the recovery and maintenance of $\mathrm{DH}$ depends on the type of support used and the angle of pedicle screw placement. While the need for such a comparison was expressed previously [17], only a few studies have addressed this topic. In the present study, we compiled patient data on the clinical efficacy of the procedure and sagittal, coronal and implant parameters before and after the surgery, to analyze the correlation between clinical efficacy and radiological outcomes. We hypothesized that different insertion trajectories of screws are associated with a significant difference in recovery of intervertebral space height, but that the comparison of clinical outcomes would show a similar improvement at follow-up. The investigation of optimal insertion trajectory of screws may help the surgeons to develop an optimal technique for patients with spondylolisthesis and to better manage subsequent rehabilitation.

\section{METHODS}

\section{Study Design and Consent}

The study was conducted in accordance with the Declaration of Helsinki (as revised in 2013). The study was approved by ethics board of Tongji Hospital of Tongji University (No.: K-2017-008) and individual consent for this retrospective analysis was waived.

\section{Inclusion and Exclusion Criteria}

The inclusion criteria were: (1) single-segment decompression and fusion with internal fixation performed for DLS; (2) age $\geq 18$ and $\leq 75$ years; (3) availability of clinical data, including general patient information [age, sex, body mass index (BMI), surgical fusion segment, spondylolisthesis segment]; lumbar $\mathrm{X}$-ray; computed tomography (CT) before and after the operation; Oswestry Disability Index (ODI) and the Visual Analogue Scale (VAS) scores for lower back and leg pain; and (4) patient followed up for at least 6 months.

The exclusion criteria were: (1) failure to complete follow-up; (2) failure of internal fixation and second operation; (3) multi-segmental operation; (4) condition combined with serious spinal deformity, tumor, fracture or other spinal diseases; and (5) condition combined with Parkinson's disease, cerebrovascular accident or other diseases.

\section{Follow-Up Method}

The patients were followed up for at least 6 months before and after the surgery. Both standard anteroposterior and lateral X-rays of 
the lumbar spine and CT of the lumbar spine had been performed. The preoperative and postoperative X-ray and CT imaging data of patients who met the inclusion and exclusion criteria and who had underwent single-segment decompression and fusion with internal fixation from May 2015 to October 2019 were obtained. The sagittal, coronal and implant parameters [anterior disc height (ADH),

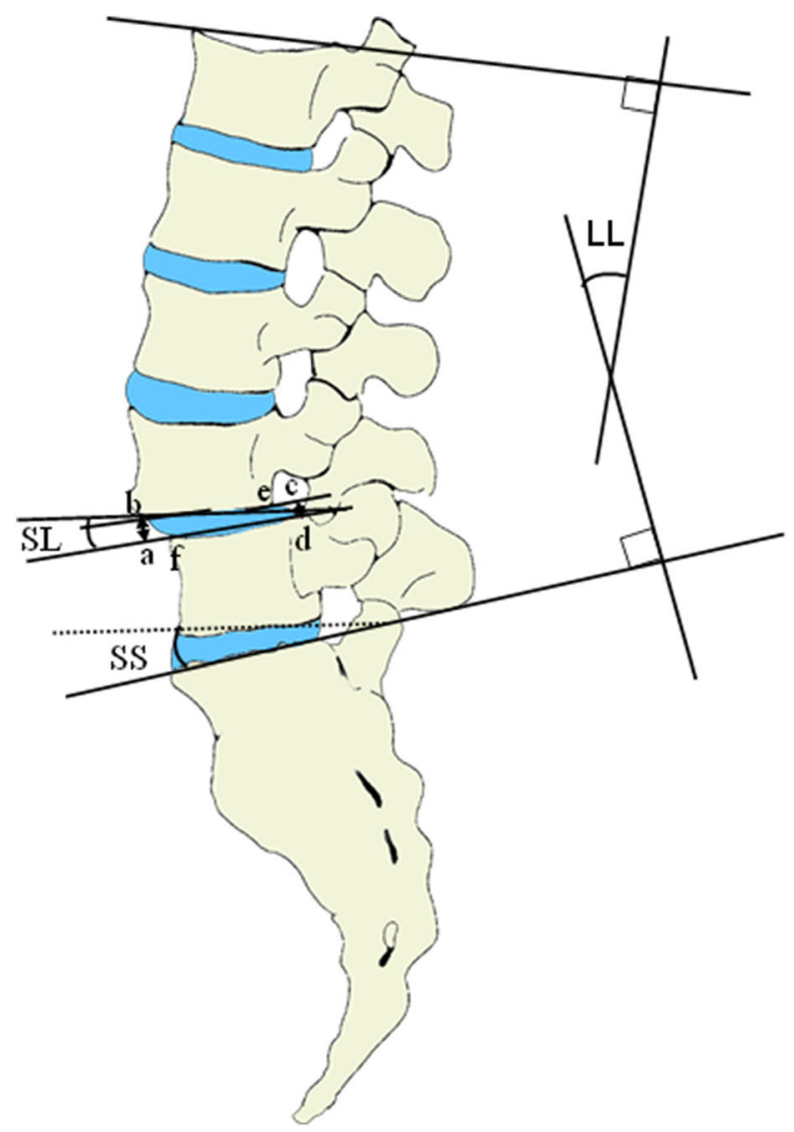

Fig. 1 Measurement of lumbar sagittal parameters. $a b$ indicates the anterior disk height $(\mathrm{ADH}), c d$ indicates the posterior disk height $(\mathrm{PDH})$. Disc height $(\mathrm{DH})$ was measured as the minimum distance $(\mathrm{mm})$ between the superior and inferior endplates of adjacent vertebral bodies at the anterior and posterior surfaces. $\mathrm{DH}$ was calculated as $(\mathrm{ADH}+\mathrm{PDH}) / 2 . d f$ is the anteroposterior width of the lower vertebral body. Slip $(\%)=c e / d f$. SL Angle between the superior and inferior endplates of the adjacent superior and inferior vertebral bodies, respectively, $L L$ angle between the superior $S 1$ and L1 endplates. $S S$ is the angle subtended by a line drawn along the endplate of the sacrum and a horizontal reference line extended from the posterior/anterior superior corner of $\mathrm{S} 1$ posterior disc height (PDH), angle of pedicle screw inclination, vertebral height, lordosis angle, cage angle, among others] were measured by spinal surgeons (Figs. 1, 2). Demographic characteristics, including age, gender and BMI, were collected for all patients at the beginning of this study. Patient-reported outcomes, including the ODI and VAS scores, were collected during follow-up. For the ODI, a lower score corresponds with better function; for the VAS, a higher score indicates worse symptoms.

\section{Measurement of Lumbar Sagittal and Coronal Parameters}

The radiological analysis included the measurement and evaluation of pre-and post-operative images using a measurement software developed with the help of custom Matlab programs (The Math Works, Natick, MA, USA). As shown in Fig. 1, $\mathrm{ADH}, \mathrm{PDH}$, lordosis angle in the fusion segment and adjacent intervertebral space were measured pre-operatively and at the final follow-up visit, as was lumbar lordosis (LL). DH was calculated as the average of the $\mathrm{ADH}$ and $\mathrm{PDH}$ at every lumbar fusion segment. To evaluate the restoration of $\mathrm{DH}$, one parameter other than $\mathrm{DH}$ was calculated. $\Delta \mathrm{DH}$ was defined as (post-operation fusion $\mathrm{DH}$ ) - (preoperation fusion DH). Similarly, $\Delta \mathrm{LL}$ was defined as (post-operation LL) - (pre-operation LL). All length parameters were in millimeters, unless otherwise specified. Slip (\%) was measured as a percentage of the distance from the posterior border of the caudal vertebra to the posterior border of the rostral vertebra. $\Delta$ Thillard $(\%)=$ (pre-operation - post-operation slip percentage)/(pre-operation slip percentage) $\times 100$.

\section{Measurement of Implant Parameters}

Pedicle screw parameters were measured as shown in Fig. 2A. The pedicle screw angle is the angle between the pedicle screw and the upper endplate of the vertebral body, and $\alpha$ and $\beta$ are the upper and lower pedicle screw angles, respectively.

Cage parameters were measured as shown in Fig. 2B, C. Our preliminary study [18] on how the positioning variables of the cage contribute to adjacent facet joint degeneration showed 

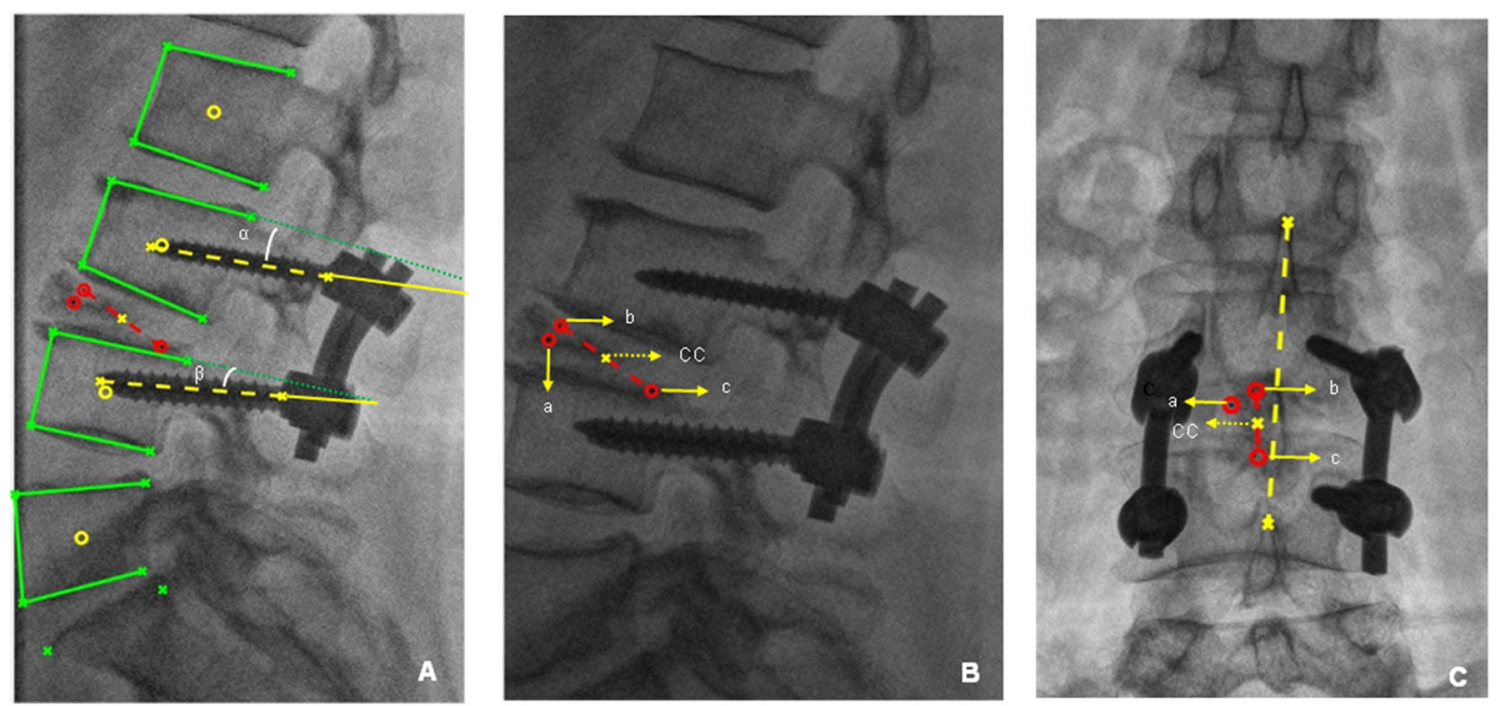

Fig. 2 Radiographic measurement of implant parameters. a Identification of vertebral contours and implant. The pedicle screw angle is the angle between the pedicle screw and the upper endplate of the vertebral body. $\alpha, \beta$ Upper and lower pedicle screw angles, respectively. b, c Sagittal and coronal measurements of cage parameters. $a, b, c$ Three feature points of cage, $C C$ center point of cage. The

that the method of measurement of cage parameters (and not explained here).

CT scans were performed as a diagnostic tool for the analysis of interbody fusion. The degree of interbody fusion was classified according to the criteria outlined by Siepe et al. [19]. The images were evaluated by two independent and highly specialized spinal surgeons with distinct expertise in the evaluation of spinal pathologies. To be confirmed as a solid fusion, both surgeons had to independently confirm either a first- or a second-degree fusion according to the classification system [19]. A third- (incomplete signs of fusion) or a fourth-degree interbody fusion assessment was defined and recorded as a nonunion/pseudarthrosis. We used the intraclass correlation coefficient (ICC) to evaluate the consistency of clinical evaluators to postoperative fusion outcomes. If the evaluation results were inconsistent, another senior physician was called in to provide an evaluation; the evaluation by the third senior physician prevailed. The grade of degeneration of anterior and posterior distances, upper and lower distances between CC and the intervertebral space can be measured. The central axis of the cage is the line between $a$ and CC, and the angle between the cage and the lower endplate can be measured. $y_{1} y_{2}$ is the line of the spinous process vertex, and the distance between CC and $y_{1} y_{2}$ can be measured. The angle between the cage axis and $y_{1} y_{2}$ can be measured

spondylolisthesis was evaluated according to the Meyerding grading.

\section{Statistical Analysis}

All data were imported into SPSS version 21.0 (IBM, Armonk, NY, USA) for statistical analysis. Continuous variables were presented as means and standard errors. Paired $t$ tests were used to analyze the differences before and after the operation. A normal test was conducted to determine whether the dependent variable was normally distributed or not. The outcomes of sagittal, coronal and implant parameter measurements were analyzed using univariate and multivariate regression analyses. The correlation coefficient $r$ was used to describe the degree of linear correlation between the variables. The VAS and ODI data, measured pre-operatively, 2 weeks after the operation and at the final follow-up ( $\geq 6$ months after the operation) were tested by one-way analysis of variance with 
repeated measures, and a two-sided $P$ value $<0.05$ was considered to be significant.

\section{RESULTS}

\section{Patient Demographics and Clinical Outcomes}

From May 2015 to October 2019, a total of 50 patients were initially enrolled, two patients were lost to follow-up after 6 months, 48 patients (17 men, 31 women) were eventually enrolled, and the follow-up rate was $96 \%$. The mean age of the 48 patients analyzed was 63.04 (range 53.34-72.74) years, and the average BMI was 25.18 (range 21.7-28.66) $\left(\mathrm{kg} / \mathrm{m}^{2}\right)$ (Table 1). The average follow-up duration for all patients was $9.16( \pm \mathrm{SE}) \pm 2.21$ (range 6.0-17.0)

Table 1 Descriptive summary of the patient cohort

\begin{tabular}{ll}
\hline Descriptive variables & $\begin{array}{l}\text { No. of patients }(\boldsymbol{N}=\mathbf{4 8} \\
\text { patients })\end{array}$ \\
\hline $\begin{array}{l}\text { Age (years), mean } \pm \text { SE } \\
\text { Sex (male/female), } n\end{array}$ & $17.04 \pm 9.70$ \\
$\quad(\%)$ & \\
BMI $\left(\mathrm{kg} / \mathrm{m}^{2}\right)$, mean \pm & $25.18 \pm 3.48) / 31(64.6 \%)$ \\
SE & \\
Fusion segment, $n(\%)$ & \\
L3/4 & $1(2.1)$ \\
L4/5 & $42(87.5)$ \\
L5/S1 & $5(10.4)$ \\
Slip vertebrae, $n(\%)$ & \\
L3 & $1(2.1)$ \\
L4 & $42(87.5)$ \\
L5 & $5(10.4)$ \\
Fusion rate (\%) & $83.3 \%(40 / 48$ patients) \\
\hline
\end{tabular}

Values in table are reported as the mean $\pm S E$ or as the number of patients with the percentage in parenthese, unless indicated otherwise

$S E$ Standard error, $B M I$ body mass index, $L$ lumbar vertebra, $L 5 / S 1$ lumbosacral joint months. Spondylolisthesis in L3, L4 and L5 was observed in 1, 42 and 5 cases, respectively. All patients underwent single-level fusions; one patient had internal fixation at L3/4, 42 patients had internal fixation at L4/5 and five patients had internal fixation at the lumbosacral joint (L5/S1). The data of the two surgeons showed a high consistency [ICC $=0.815$ (range 0.712, $0.884)$ ], and there was no need for a third party to evaluate. The fusion rate was $83.3 \%(40 / 48$ cases). At the final follow-up, no post-operative symptoms were observed among all patients with adequate fusion; among the eight patients with inadequate fusion, five had mild persistent low back pain, and adjacent segmental degeneration (ASD) occurred in the other three. There was no implant failure or displacement, and no revision surgery was required. No major complications, such as neural and vascular injury or deep wound infection, occurred.

\section{Radiographical Outcomes}

The sagittal sequence parameters of the lumbar spine were compared pre-operation and at 6 months post-operation, and the results are summarized in Table 2 . The values at these two time points were not significantly different for the angle between the superior and inferior endplates of the adjacent superior and inferior vertebral bodies (SL), PDH and LL of the fusion segment; however, compared to pre-operation, the $\mathrm{ADH}$ and the $\mathrm{DH}$ of the fusion segment increased, and the Meyerding grade and Taillard score had decreased significantly at 6 months post-operation $(p<0.05)$.

\section{Analysis of the Effect of Decompression and Fusion with Internal Fixation in the Treatment of DLS}

As shown in Table 3, the VAS scores of low back pain 2 weeks after operation were significantly improved compared to those before the operation $(p<0.05)$. At the final follow-up, the VAS and ODI showed significant improvement compared with pre-operative values in both groups $(p<0.05)$. The results of the analysis of 
Table 2 Comparisons of sagittal sequence parameters of the lumbar spine at pre-operation and 6 months post-operation

\begin{tabular}{lcllc}
\hline Sagittal sequence parameters & Pre-operation & Final follow-up post-operation & $\boldsymbol{P}$ value & \multicolumn{1}{l}{ Differences } \\
\hline $\mathrm{SL}\left(^{\circ}\right)$ & $14.29 \pm 8.17$ & $15.16 \pm 6.58$ & 0.274 & $0.87 \pm 5.57$ \\
$\mathrm{ADH}(\mathrm{mm})$ & $14.69 \pm 6.75$ & $17.36 \pm 6.81$ & $0.009^{*}$ & $2.67 \pm 6.90$ \\
$\mathrm{PDH}(\mathrm{mm})$ & $9.20 \pm 4.52$ & $10.42 \pm 4.36$ & 0.105 & $1.22 \pm 5.20$ \\
$\mathrm{DH}(\mathrm{mm})$ & $11.95 \pm 5.25$ & $13.89 \pm 5.19$ & $0.020^{*}$ & $1.94 \pm 5.70$ \\
$\mathrm{LL}\left(^{\circ}\right)$ & $41.86 \pm 12.45$ & $43.20 \pm 11.49$ & 0.361 & $1.34 \pm 10.24$ \\
Meyerding grade & $1.10 \pm 0.30$ & $0.62 \pm 0.49$ & $0.000^{*}$ & $-0.48 \pm 0.54$ \\
Taillard score & $14.76 \pm 11.59$ & $4.49 \pm 0.49$ & $0.000^{*}$ & $-10.27 \pm 9.68$ \\
\hline
\end{tabular}

Values are presented as the mean $\pm \mathrm{SE}$

$S L$ Segment lordosis, $A D H$ anterior disc height, $P D H$ posterior disc height, $D H$ disc height, $L L$ lumbar lordosis

*Values are statistically significant at $P<0.05$

Table 3 Changes in Oswestry Disability Index and Visual Analogue Scale scores

\begin{tabular}{lccc}
\hline $\begin{array}{l}\text { Oswestry Disability Index and the Visual } \\
\text { Analogue Scale }\end{array}$ & $\begin{array}{l}\text { Pre- } \\
\text { operation }\end{array}$ & $\begin{array}{l}\text { Two weeks post- } \\
\text { operation }\end{array}$ & $\begin{array}{l}\text { Final follow-up post- } \\
\text { operation }\end{array}$ \\
\hline VAS score & $6.92 \pm 0.97$ & $3.80 \pm 1.16^{*}$ & $0.70 \pm 0.79^{* \#}$ \\
ODI score & $63.52 \pm 5.88$ & $33.10 \pm 6.67^{*}$ & $15.20 \pm 3.55^{* \#}$ \\
\hline
\end{tabular}

Values in table are presented as the mean \pm standard deviation

*Significant difference with pre-operation value at $P<0.05$; ${ }^{*}$ significant difference wtih 2 weeks post-operation value at $P<0.05$

$V A S$ Visual Analogue Scale, ODI Oswestry Disability Index

the improvement rates of lumbar spondylolisthesis (LS) after the surgical treatment are shown in Fig. 3. The correction rate of the standard score was $>80 \%$ in 17 cases $(35.4 \%)$ and $>20 \%$ in 43 cases $(87.5 \%)$, suggesting that vertebral spondylolisthesis improved to varying degrees.

\section{Correlations Between the Changes in Deformity Parameters, Sagittal Parameters and Pedicle Screw Angles}

Table 4 shows that the restoration of PDH and $\mathrm{DH}$ was correlated with the lower pedicle angle $(r=-0.296, P=0.041 ; r=-0.306, P=0.035$, respectively) at 6 months after surgery for DLS. There was a significant correlation between upper and lower pedicle screw angles $(P<0.01)$. The change in VAS was significantly correlated with the lower pedicle screw angles. Other improvements in sagittal parameters showed no significant correlation with the upper and lower pedicle screw angles.

Test of normality was performed for the dependent variable of $\triangle \mathrm{ADH}$ and $\triangle \mathrm{PDH}$. Because of the small sample size of our study, results of the Shapiro-Wilk test were more suitable as reference for the test of normality. According to Table 5, the statistical magnitudes of $\triangle \mathrm{ADH}$ and $\triangle \mathrm{PDH}$ were 0.161 and 0.493 , respectively $(P>0.05)$, indicating that the $\triangle \mathrm{ADH}$ and $\triangle \mathrm{PDH}$ are normal variables.

\section{Multivariate Regression Analysis}

The clinical efficacy index, lumbar spine parameters and pedicle screw angles were analyzed using backward stepwise regression. The 


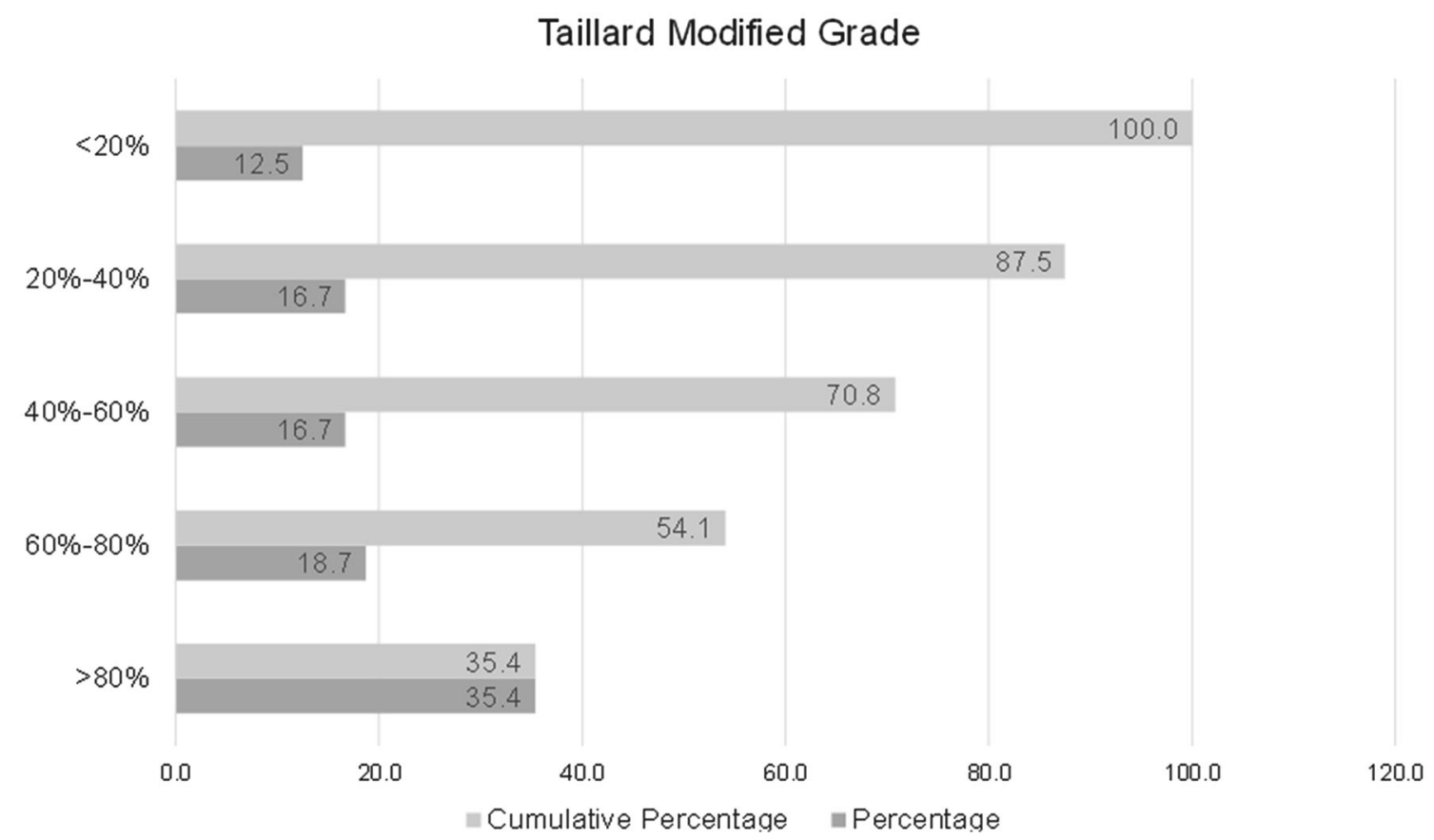

Fig. 3 Results of the analysis of the improvement rates of degenerative lumbar spondylolisthesis after surgical treatment

Table 4 Pearson correlation analysis of the upper and lower pedicle screw angles and dependent variables

\begin{tabular}{llllllllll}
\hline Variables & \multicolumn{2}{l}{ Dependent $(\mathbf{D})$ variables } \\
\cline { 2 - 9 } & $\boldsymbol{\alpha}$ & $\boldsymbol{\beta}$ & $\Delta \mathbf{A D H}$ & $\Delta \mathbf{P D H}$ & $\Delta \mathbf{D H}$ & $\Delta \mathbf{S S}$ & $\Delta \mathbf{L L}$ & $\Delta$ VAS & $\Delta$ ODI \\
\hline$\alpha$ & & & & & & & & & \\
$r$ & 1 & $0.449^{* *}$ & -0.002 & -0.103 & -0.187 & -0.081 & -0.086 & -0.260 & -0.201 \\
$P$ value & - & 0.001 & 0.989 & 0.487 & 0.203 & 0.582 & 0.559 & 0.075 & 0.170 \\
$\beta$ & & & & & & & & & \\
$r$ & $0.449^{* *}$ & 1 & -0.131 & $-0.296^{*}$ & $-0.306^{*}$ & 0.239 & -0.145 & $-0.297^{*}$ & -0.161 \\
$P$ value & 0.001 & - & 0.374 & 0.041 & 0.035 & 0.102 & 0.326 & 0.041 & 0.276 \\
\hline
\end{tabular}

$\alpha$ Upper pedicle screw angle, $\beta$ lower pedicle screw angle, $\triangle \mathrm{ADH}=$ (post-operation fusion $\mathrm{ADH})-($ pre-operation fusion $\mathrm{ADH}), \Delta \mathrm{PDH}=($ post-operation fusion $\mathrm{PDH})-($ pre-operation fusion $\mathrm{PDH}), \Delta \mathrm{DH}=($ post-operation fusion $\mathrm{DH})-$ (pre-operation fusion DH), $\Delta \mathrm{LL}=$ (post-operation LL) - (pre-operation LL); $\Delta S S$ (sacral slope) $=$ (post-operation SS) (pre-operation SS), $\triangle \mathrm{VAS}=($ post-operation VAS $)-($ pre-operation VAS $), \Delta \mathrm{ODI}=($ post-operation ODI $)-($ pre-operation ODI)

${ }^{*}$ Statistically significant Pearson correlation coefficient $(r)$ at $P<0.05$

independent variables included pre-and pos-toperative fusion segment $\mathrm{ADH}, \mathrm{PDH}, \mathrm{DH}$ and SL; the lumbar sagittal parameters LL and SS; and the changes in these parameters. The upper and lower pedicle screw inclinations were also used as independent variables in the multiple regression analysis. Table 6 shows the regression coefficients for each predictive variable adjusted 
Table 5 Output of the test for normality

\begin{tabular}{llll}
\hline $\begin{array}{l}\text { Dependent } \\
\text { variable }\end{array}$ & \multicolumn{2}{l}{ Shapiro-Wilk test } \\
\cline { 2 - 4 } & Statistic & $d f$ & $\begin{array}{l}\text { Statistical } \\
\text { magnitude }\end{array}$ \\
\hline$\Delta \mathrm{ADH}$ & 0.965 & 48 & 0.161 \\
$\Delta \mathrm{PDH}$ & 0.978 & 48 & 0.493 \\
\hline
\end{tabular}

by the fitting. The change in $\mathrm{DH}$ and fusion segment SL was positively correlated with the change in ODI and VAS scores, respectively. The upper pedicle screw angle was significantly negatively correlated with the change in VAS score. The reduction of the upper pedicle screw angle per unit reduced the increased value of VAS by 0.041 units. These results indicated that the preo-perative lumbar spine parameters did not significantly affect the clinical outcomes of surgery for DLS, except for the percentage of change rate $(\%)$.

A significant negative correlation was observed between the angle of the superior pedicle screw and the anterior height recovery of the fused segment. The reduction of the upper pedicle screw angle per unit reduced the anterior DH recovery value by 0.323 units. Moreover, a significant negative correlation was observed between the angle of the inferior pedicle screw and the recovery of posterior height. The reduction of the lower pedicle screw angle per unit reduced the recovery value of PDH by 0.196 units.

Together, these results indicate that within a certain range, decompression and fusion with internal fixation can correct high or low anterior and posterior heights of the intervertebral space and can recover the lordosis angle with high slippage. Also, the results of the multivariate stepwise regression analysis showed a correlation between the pedicle screw angle and post-operative height recovery in the intervertebral space. A larger downward inclination angle between the upper and lower pedicle screws was conducive to the recovery of the anterior and posterior height of the intervertebral space.

\section{DISCUSSION}

Several factors affect the post-operative clinical efficacy of DLS. Lumbar instability, defined as the loss of the ability of the spine under physiological stress to maintain its pattern of displacement [20], is one such factor that can adversely affect fusion rates. Abnormal sagittal lumbar alignment after lumbar fusion may

Table 6 Regression coefficients for each predictive variable adjusted by the fitting

\begin{tabular}{|c|c|c|c|c|c|c|c|c|c|c|}
\hline \multirow[t]{2}{*}{ D-Variables } & \multicolumn{2}{|c|}{$\Delta$ Taillard (\%) } & \multicolumn{3}{|l|}{$\Delta$ VAS } & $\Delta O D I$ & \multicolumn{2}{|l|}{$\Delta \mathrm{ADH}$} & \multicolumn{2}{|l|}{$\Delta \mathbf{P D H}$} \\
\hline & Coe & $p$ value & Coe & $p$ value & Coe & $\overline{p \text { value }}$ & Coe & $p$ value & Coe & $\overline{p \text { value }}$ \\
\hline \multicolumn{11}{|l|}{ Variables } \\
\hline$\alpha$ & - & - & -0.041 & $0.038^{*}$ & - & - & -0.323 & $0.035^{*}$ & - & - \\
\hline$\beta$ & - & - & - & - & - & - & - & - & -0.196 & $0.032^{*}$ \\
\hline preADH & - & - & - & - & 0.233 & 0.092 & -0.537 & $<0.01^{*}$ & - & - \\
\hline prePDH & 26.509 & $0.001^{*}$ & - & - & - & - & - & - & -0.671 & $<0.01^{*}$ \\
\hline PostLL & 6.080 & $0.031^{*}$ & - & - & - & - & - & - & - & - \\
\hline$\Delta \mathrm{SL}$ & -20.358 & $0.001^{*}$ & 0.051 & $0.011^{*}$ & - & - & - & - & - & - \\
\hline$\Delta \mathrm{DH}$ & - & - & - & - & 0.338 & $0.041^{*}$ & - & - & - & - \\
\hline
\end{tabular}

$\Delta$ Thailard $(\%)=($ pre-operation - post-operation slip percentage $) /($ pre-operation slip percentage $) \times 100$, Coecoefficient $\Delta \mathrm{SL}=$ (post-operation fusion SL) $-($ pre-operation fusion $\mathrm{SL})$; see footnote for Table 4 for other definitions *Values are statistically significant at $P<0.05$ 
aggravate ASD, leading to higher energy requirements for standing and ambulating, resulting in early fatigue, intolerance to standing and walking with compensation through other joints [21]. In other words, good postoperative functional results largely depend on the restoration of the normal spinal sagittal sequence and stability during operation $[22,23]$. Moderate evidence indicates that decompression alone may be a feasible treatment option, with lower surgical morbidity and outcomes similar to those of fusion when performed in a select population with low-grade slip [24, 25]. Similarly, the addition of interbody fusion may be best suited to a subset of patients with high-grade DLS [26-28], although this is yet to be established. Data on sagittal, coronal and implant parameters were collected in this study. However, due to space limitation, only the data on pedicle screw inclination and intervertebral space height recovery were analyzed.

While pedicle screw placement is generally considered the most popular method in lumbar spinal instrumentation for achieving successful fusion, successful outcomes are largely dependent on obtaining a solid fusion and recreating the normal DH. Pedicle screw fixation and intervertebral fusion restore intervertebral displacement, maintain the balance between the spine and the pelvis, provide immediate spinal stability, relieve symptoms, prevent progressive slippage and increase the fusion rate [29]. For the lumbar vertebrae affected by osteoporosis, pedicle screws inserted using the Krag (screw pointed toward the superior endplate) and Caudad (screw pointed toward the caudal part of the body) trajectory techniques have been found to provide better biomechanical stability than those inserted parallel to the superior endplate using the Magerl technique [16]. Although the Caudad approach is not close to the upper and lower endplates, the trajectory is close to the cortical bone of the upper and lower walls of the pedicle, and it also runs through the middle and rear of the vertebral body, pointing forward and downwards, increasing the area of contact with cortical bone, which may be the reason why the Caudad approach can obtain better biomechanical stability [16]. Our findings imply that the Caudad insertion of pedicle screws facilitated interbody distraction during the restoration of spondylolisthetic vertebrae. A larger downward inclination angle of the upper and lower pedicle screws was conducive to the recovery of the anterior and posterior height of the intervertebral space. These findings are consistent with those of a previous biomechanical study reporting significantly higher biomechanical strength for pedicle screws inserted in osteoporotic lumbar vertebrae using the Caudad trajectory compared to those inserted using the Krag trajectory, especially during early fixation [30]. One explanation for this finding might be that when the screws are inserted into the pedicle of an vertebral arch angulated, the holding force will be greater than when they are inserted at $0^{\circ}$ [31]. The holding force is increased as the angle is augmentated. Another factor is that when the cage was used as the fulcrum, compression fixation with the upper pedicle screw in the caudal approach resulted in greater longitudinal pressure, and the recovery of the corresponding pre-intervertebral space height was increased compared with that with the pedicle screw parallel to the upper endplate (Fig. 4a). Similarly, the longitudinal pressure required for compression fixation with lower pedicle screws placed in the caudal approach was correspondingly reduced, which is conducive to maintain the $\mathrm{PDH}$ after the operation compared to pedicle screws placed parallel to the upper endplate (Fig. 4b). However, additional biomechanical studies are needed to confirm this speculation and verify whether the results of the present study can be applied to patients with LS with normal bone mineral density.

Regarding clinical efficacy, the results of this study showed significantly improved VAS scores for low back and leg pain and improved ODI scores at 6 months after decompression and fusion with internal fixation compared with those before the operation. Also, vertebral spondylolisthesis was reduced to varying degrees. Multiple regression analysis indicated that the change in $\mathrm{DH}$ and fusion SL was positively correlated with changes in ODI and VAS scores, respectively, consistent with the findings of previous studies [32]. While previous studies 


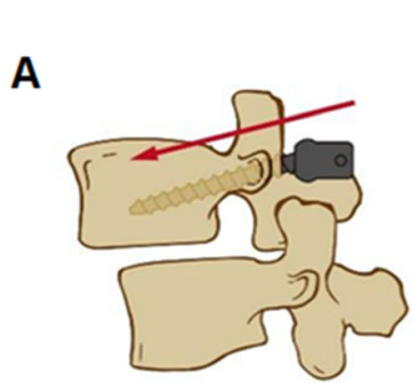

(1)

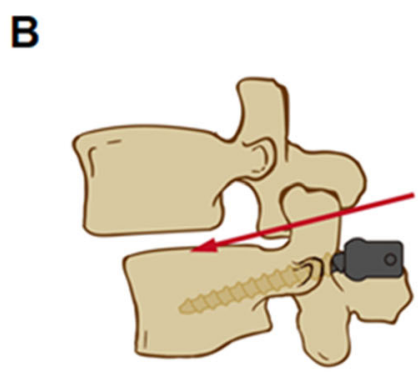

(1)

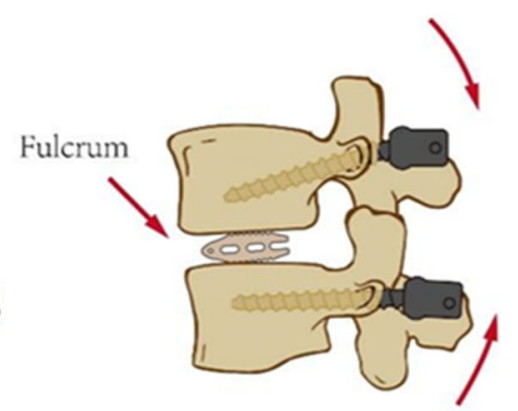

(2)

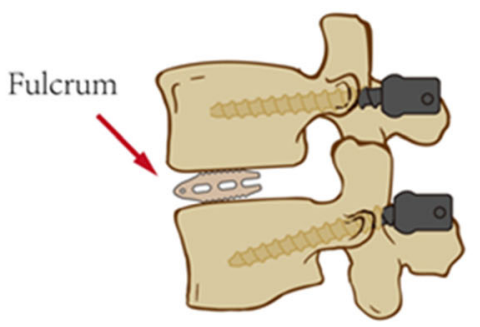

(2)

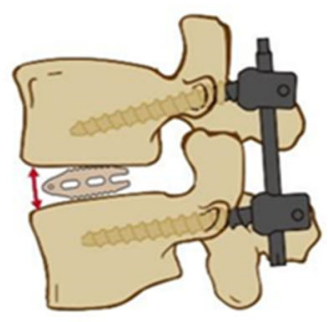

(3)

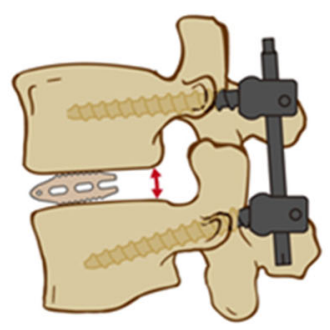

(3)
Fig. 4 Schematic images of the restoration of intervertebral disc height during surgical procedures. a The upper pedicle screw in the Caudad insertion. (1) Insertion of upper pedicle screws is moderately downward instead of parallel to the upper vertebral endplate in the sagittal plane, (2) a cage is placed as a fulcrum and posterior instrumentation is compressed, (3) a satisfactory and higher

observed a change in sagittal balance at least 2 years after surgery $[33,34]$, we observed similar outcomes in a mean follow-up period of only 6 months. This improvement might be due to better distraction of the intervertebral space, which maintains the stability of the lumbar spine and is conducive to the improvement of clinical effects. However, biomechanical and clinical indicators are needed for further verification and comparative analysis. Feng et al. [34, 35] and Park et al. [36] reported that DH recovery was positively correlated with the increase in LL and that the full restoration of intervertebral space height was conducive to LL recovery and reduction of intervertebral disc pressure at the fusion segment. Furthermore, anterior disc height is obtained after pedicle screw fixation. b The lower pedicle screw in the Caudad insertion. (1) Insertion of lower pedicle screws is moderately downward instead of parallel to the upper vertebral endplate in the sagittal plane, (2) a cage is placed as a fulcrum and posterior instrumentation is compressed, (3) a satisfactory posterior disc height is maintained after pedicle screw fixation

the recovery of intervertebral space height and the tension bending effect caused by lumbar ligament tightening were also conducive to the prevention of ASD. In contrast, our study results showed no significant correlation between LL and $\mathrm{DH}$, which may be due to insufficient follow-up time and recovery of intervertebral space height. To reduce variability and fully assess the efficacy of the technique, further studies must evaluate clinical and radiological outcomes in larger study populations and conduct long term follow-up together with the collection of clinical effect indexes and the measurement of sagittal parameters.

The potential limitations of this study include its retrospective design and the 
resultant reduced level of evidence. In addition, a small sample size and short follow-up time may have led to bias in the results of the data collection. However, this is one of the few studies to have investigated the relationship between pedicle screw inclination and restoration of intervertebral space height. Although the results of this study suggest a negative correlation between pedicle screw inclination and post-operative DH recovery, the potential advantages of a larger pedicle screw inclination in terms of post-operative clinical efficacy and complications have not yet been validated. Therefore, future research should focus on the assessment of pedicle screw angle, including in vitro analysis and prospective studies.

\section{CONCLUSIONS}

In a retrospective study involving 48 patients who underwent single-segment decompression and fusion with internal fixation for DLS at our institution with at least 6 months of radiographical and clinical follow-up, we determined that the Caudad insertion trajectory technique of pedicle screws is an ideal alternative for the treatment of DLS.

\section{ACKNOWLEDGEMENTS}

Funding. This study and the journal's Rapid Service Fee were funded by research grants from the National Natural Science Foundation of China (No. 81873774), Shanghai Rising-Star Program (No. 18QA1403800), Shanghai Excellent Young Medical Talents Training Program (No. 2018YQ22) and Shanghai Municipal Health Commission Program (No. 201940244).

Editorial Assistance. We would like to thank Editage (www.editage.cn) for English language editing.

Authorship. All named authors meet the International Committee of Medical Journal Editors (ICMJE) criteria for authorship for this article, take responsibility for the integrity of the work as a whole and have given their approval for this version to be published.

Author contributions. (Conception and design: TY Tsai, LM Cheng, G Li, Y Yu; administrative support: LM Cheng, Y Yu, Z Zeng, B $\mathrm{Ma}, \mathrm{N}$ Xie; provision of study materials or patients: LM Cheng, Y Yu, Z Zeng, B Ma, N Xie; collection and assembly of data: X Xi, FP Li, C Wang; data analysis and interpretation: $\mathrm{X} \mathrm{Xi}, \mathrm{Z}$ Zeng, $\mathrm{FP} \mathrm{Li}, \mathrm{Y} \mathrm{Yu}$; manuscript writing: All authors; final approval of manuscript: all authors.

Prior Presentation. The results have been presented in an oral presentation at the 13th Congress of Chinese Association of Orthopaedic Surgeons (CAOS2021), 20-23 May 2021, Chengdu, China.

Compliance with Ethics Guidelines. The study was conducted according to the Declaration of Helsinki (as revised in 2013). The study was approved by ethics board of Tongji Hospital of Tongji University (No.: K-2017-008) and individual consent for this retrospective analysis was waived.

Disclosures. Xin Xi, Zhili Zeng, Fuping Li, Cong Wang, Bin Ma, Ning Xie, Tsung-Yuan Tsai, Guoan Li, Yan Yu and Liming Cheng have nothing to disclose.

Data Availability. The datasets generated and/or analyzed during the current study are not publicly available due to the data is confidential patient data but are available from the corresponding author on reasonable request.

Open Access. This article is licensed under a Creative Commons Attribution-NonCommercial 4.0 International License, which permits any non-commercial use, sharing, adaptation, distribution and reproduction in any medium or format, as long as you give appropriate credit to the original author(s) and the source, provide a link to the Creative Commons licence, and indicate if changes were made. The images or other third party material in this article are included in the article's Creative Commons 
licence, unless indicated otherwise in a credit line to the material. If material is not included in the article's Creative Commons licence and your intended use is not permitted by statutory regulation or exceeds the permitted use, you will need to obtain permission directly from the copyright holder. To view a copy of this licence, visit http://creativecommons.org/licenses/by$\mathrm{nc} / 4.0 /$.

\section{REFERENCES}

1. Lian XF, Hou TS, Xu JG, et al. Posterior lumbar interbody fusion for aged patients with degenerative spondylolisthesis: is intentional surgical reduction essential? Spine J. 2013;13(10):1183-9.

2. Audat ZM, Darwish F, Al Barbarawi MM, et al. Surgical management of low-grade isthmic spondylolisthesis; a randomized controlled study of the surgical fixation with and without reduction. Scoliosis. 2011;6(1):14.

3. Fan GX, Wang DD, Wu XB, et al. Minimally invasive transforaminal lumbar interbody fusion for spondylolisthesis: reduction fusion vs. in-situ fusion. J Spinal Surg. 2016;14(6):335-9.

4. France JC, Yaszemski MJ, Lauerman WC, et al. A randomized prospective study of posterolateral lumbar fusion: outcomes with and without pedicle screw instrumentation. Spine. 1999;24(6):553-60.

5. Bourghli A, Aunoble S, Reebye O, et al. Correlation of clinical outcome and spinopelvic sagittal alignment after surgical treatment of low-grade isthmic spondylolisthesis. Eur Spine J. 2011;20(Suppl 5): 663-8.

6. Tay KS, Bassi A, Yeo $\mathrm{W}$, et al. Intraoperative reduction does not result in better outcomes in low-grade lumbar spondylolisthesis with neurogenic symptoms after minimally invasive transforaminal lumbar interbody fusion-a 5-year follow-up study. Spine J. 2016;16(2):182-90.

7. Stonecipher T, Wright S. Posterior lumbar interbody fusion with facet-screw fixation. Spine. 1989;14(4):468-71.

8. West JL, Bradford DS, Ogilvie JW. Results of spinal arthrodesis with pedicle screw-plate fixation. J Bone Jt Surg Am. 1991;73(8):1179-84.

9. Röllinghoff M, Schlüter-Brust K, Groos D, et al. Midrange outcomes in 64 consecutive cases of multilevel fusion for degenerative diseases of the lumbar spine. Orthop Rev (Pavia). 2010;2(1):e3.

10. Wu ZX, Gong FT, Liu L, et al. A comparative study on screw loosening in osteoporotic lumbar spine fusion between expandable and conventional pedicle screws. Arch Orthop Trauma Surg. 2012;132(4):471-6.

11. Galbusera F, Volkheimer D, Reitmaier S, et al. Pedicle screw loosening: a clinically relevant complication? Eur Spine J. 2015;24(5):1005-16.

12. Kim JB, Park SW, Lee YS, et al. The effects of spinopelvic parameters and paraspinal muscle degeneration on S1 screw loosening. J Korean Neurosurg Soc. 2015;58(4):357-62.

13. Cardoso MJ, Dmitriev AE, Helgeson M, et al. Does superior-segment facet violation or laminectomy destabilize the adjacent level in lumbar transpedicular fixation? An in vitro human cadaveric assessment. Spine (Phila Pa 1976). 2008;33(26): 2868-73.

14. Kim HJ, Chun HJ, Kang KT, et al. The biomechanical effect of pedicle screws' insertion angle and position on the superior adjacent segment in 1 segment lumbar fusion. Spine (Phila $\mathrm{Pa}$ 1976). 2012;37(19):1637-44.

15. Bokov A, Bulkin A, Aleynik A, et al. Pedicle screws loosening in patients with degenerative diseases of the lumbar spine: potential risk factors and relative contribution. Glob Spine J. 2019;9(1):55-61.

16. Yuan Q, Xing YG, Tao JF, et al. The direction of pedicle screws in osteoporotic lumbar vertebrae on stability of internal fixation. Shandong Pharm. 2009;49(20):19-22.

17. Cheung KM, Zhang YG, Lu DS, et al. Reduction of disc space distraction after anterior lumbar interbody fusion with autologous iliac crest graft. Spine. 2003;28(13):1385-9.

18. Li F, Zhan $\mathrm{X}, \mathrm{Xi} \mathrm{X}$, et al. Do the positioning variables of the cage contribute to adjacent facet joint degeneration? Radiological and clinical analysis following intervertebral fusion. Ann Transl Med. 2021. https://doi.org/10.21037/atm-20-7718.

19. Siepe CJ, Stosch-Wiechert K, Heider F, et al. Anterior stand-alone fusion revisited: a prospective clinical, X-ray and CT investigation. Eur Spine J. 2015;24(4):838-51.

20. Maigne JY, Lapeyre E, Morvan G, Chatellier G. Pain immediately upon sitting down and relieved by standing up is often associated with radiologic lumbar instability or marked anterior loss of disc space. Spine (Phila Pa 1976). 2003;28(12):1327-34. 
21. Le TV, Vivas AC, Dakwar E, et al. The effect of the retroperitoneal transpsoas minimally invasive lateral interbody fusion on segmental and regional lumbar lordosis. Sci World J. 2012;2012:516706.

22. Schwab F, Dubey A, Pagala M, et al. Adult scoliosis: a health assessment analysis by SF-36. Spine (Phila Pa 1976). 2003;28(6):602-6.

23. Lazennec JY, Ramare S, Arafati N, et al. Sagittal alignment in lumbosacral fusion:relations between radiological parameters and pain. Eur Spine J. 2000;9(1):47-55.

24. Matsudaira K, Yamazaki T, Seichi A, et al. Spinal stenosis in grade I degenerative lumbar spondylolisthesis: a comparative study of outcomes following laminoplasty and laminectomy with instrumented spinal fusion. J Orthop Sci. $2005 ; 10(3): 270-6$.

25. Aihara T, Toyone T, Aoki Y, et al. Surgical management of degenerative lumbar spondylolisthesis: a comparative study of outcomes following decompression with fusion and microendoscopic decompression. J Musculoskelet Res. 2012;15(4): 1250020 .

26. Ha KY, Na KH, Shin JH, Kim KW. Comparison of posterolateral fusion with and without additional posterior lumbar interbody fusion for degenerative lumbar spondylolisthesis. J Spinal Disord Tech. 2008;21(4):229-34.

27. Ghasemi AA. Transforaminal lumbar interbody fusion versus instrumented posterolateral fusion in degenerative spondylolisthesis: an attempt to evaluate the superiority of one method over the other. Clin Neurol Neurosurg. 2016;150:1-5.

28. Challier V, Boissiere L, Obeid I, et al. One-level lumbar degenerative spondylolisthesis and posterior approach: is transforaminal lateral interbody fusion mandatory? a randomized controlled trial with 2-year follow-up. Spine (Phila $\mathrm{Pa}$ 1976). 2017;42(8):531-9.
29. Takahashi T, Hanakita J, Ohtake Y, et al. Current status of lumbar interbody fusion for degenerative spondylolisthesis. Neurol Med Chir. 2016;56(8): 476-84.

30. Yuan Q, Han XG, Han X, et al. Krag versus Caudad trajectory technique for pedicle screw insertion in osteoporotic vertebrae: biomechanical comparison and analysis. Spine (Phila Pa 1976). 2014;39(26 Spec No.):B27-35.

31. Shi K, Lei Y, Wang H, et al. Biomechanical study of pull-out force on pedicle screws of different sagittal angles. Chin J Bone Jt Injury. 2009;24(4):311-3.

32. Seuk JW, Bae J, Shin SH, Lee SH. Long-term minimum clinically important difference in health-related quality of life scores after instrumented lumbar interbody fusion for low-grade isthmic spondylolisthesis. World Neurosurg. 2018;117: e493-9.

33. Kim $\mathrm{CH}$, Chung $\mathrm{CK}$, Choi $\mathrm{Y}$, et al. Increased proportion of fusion surgery for degenerative lumbar spondylolisthesis and changes in reoperation rate: a nationwide cohort study with a minimum 5-year follow-up. Spine (Phila Pa 1976). 2019;44(5): 346-54.

34. Feng Y, Chen L, Gu Y, et al. Influence of the posterior lumbar interbody fusion on the sagittal spino-pelvic parameters in isthmic L5-S1 spondylolisthesis. J Spinal Disord Tech. 2014;27(1):e20-5.

35. Feng Y, Liang C, Yong G, et al. Restoration of the spinopelvic sagittal balance in isthmic spondylolisthesis: posterior lumbar interbody fusion may be better than posterolateral fusion. Spine J. 2015;15:1527-35.

36. Park SJ, Lee CS, Chung SS, et al. Postoperative changes in pelvic parameters and sagittal balance in adult isthmic spondylolisthesis. Neurosurgery. 2011;68(2 Suppl Operative):355-63. 